\title{
PERFIL EPIDEMIOLÓGICO DE PACIENTES ATENDIDOS NO AMBULATÓRIO DO SERVIÇO DE REFERÊNCIA NACIONAL EM FILARIOSES DO CENTRO DE PESQUISAS AGGEU MAGALHÃES, RECIFE-PE
}

Paula Fernanda Alcântara de Souza ${ }^{1}$, Eduardo Caetano Brandão Ferreira da Silval, Ana Maria Aguiar Santos ${ }^{1}$, Zulma Maria de Medeiros ${ }^{1}$, Rodrigo Luis Ferreira da Silva², Paula Alexandra dos Santos Oliveiral ${ }^{1}$ Paulo Sérgio Ramos de Araújol $^{1}$, Maria José Teixeiral e Abraham Rocha ${ }^{1}$

\section{RESUMO}

Este trabalho descreve o perfil epidemiológico dos pacientes atendidos no ambulatório do Serviço de Referência Nacional em Filarioses (SRNF), onde são desenvolvidas atividades clínicas, epidemiológicas e laboratoriais relacionadas à pesquisa e assistência aos pacientes acometidos por filarioses. As informações foram obtidas de 1.109 prontuários de pacientes atendidos no período de seis anos. Do total de prontuários analisados, foi observado que $62,8 \%$ dos indivíduos atendidos eram do gênero masculino e que $85,4 \%$ eram residentes na Região Metropolitana do Recife (RMR). A frequência de indivíduos encaminhados ao SRNF que se apresentavam microfilarêmicos e/ou antígenos positivos para filariose bancroftiana foi de $55 \%$. Tal resultado foi considerado baixo uma vez que esses indivíduos apresentavam forte indicativo clínico ou epidemiológico para infecção filarial. Esta informação evidencia a necessidade de um esforço maior no estabelecimento de parâmetros que determinem o fluxo de encaminhamento dos pacientes ao SRNF, de modo que se evite demanda desnecessária e sejam otimizados os recursos.

DESCRITORES: Filariose; assistência à saúde; perfil de saúde; acesso aos serviços de saúde.

\section{ABSTRACT}

Epidemiological profile of patients seen at in the outpatient National Reference Service for Filariasis, of Aggeu Magalhães Research Center, Recife-PE

This paper describes the epidemiological profile of patients attending the outpatient clinic of the Department of the National Reference Service for Filariasis (SRNF), where clinical, epidemiological

1 Serviço de Referência Nacional em Filarioses - Departamento de Parasitologia, Centro de Pesquisas Aggeu Magalhães, Fundação Oswaldo Cruz, Recife, PE.

2 Instituto de Ciências Biológicas da Universidade de Pernambuco, Recife, PE.

Endereço para correspondência: Paula Fernanda Alcântara de Souza. Depto. de Parasitologia, Serviço de Referência Nacional em Filarioses, Av. Moraes Rego S/N, 50670-420, Recife-PE, Brasil. E-mail: pfalcantara@cpqam.fiocruz.br 
and laboratory activities are carried out relating to research, care, investigation and monitoring of patients suffering from filariasis. Information was obtained from the medical records of 1,109 patients treated over a six year period. It was observed that most of the subjects attending came from of the Metropolitan Region of Recife (RMR) - 85.4\%,-most of whom (62.8\%.) were male. A low frequency of individuals referred to SRNF showed microfilariae or antigen positivity for lymphatic filariasis $(55 \%)$, although these patients represent strong evidence for clinical or epidemiological filarial infection. This information makes clear the need for a greater effort to establish parameters that determine the flow of referral of patients to SRNF, avoiding unnecessary demand and allowing optimization of resources.

KEY WORDS: Filariasis; delivery of health care; health profile; health services accessibility.

\section{INTRODUÇÃO}

A filariose linfática (FL) é uma doença negligenciada, de caráter debilitante e seu principal agente etiológico são os helmintos da espécie Wuchereria bancrofti (Rey, 2001; Fontes et al., 2005). De acordo com a Organização Mundial da Saúde (OMS), estima-se em cerca de 120 milhões o número de indivíduos afetados pela FL, distribuídos em 72 países tropicais e subtropicais (WHO, 2008, 2010). No continente americano, a estimativa é que existam, aproximadamente, 9 milhões de indivíduos vivendo em áreas com risco de contrair a parasitose e 720 mil infectados (OPAS, 2007).

No Brasil, até recentemente, a filariose linfática era encontrada nas cidades de Maceió-AL e Belém-PA, mas, de acordo com a Organização Pan-Americana da Saúde (OPAS), a infecção no estado de Alagoas se encontra em etapa de pré-eliminação e, no Pará, a transmissão parece ter sido interrompida. $\mathrm{Na}$ atualidade, a Região Metropolitana do Recife (RMR)-PE é o principal foco da doença no território brasileiro, com uma população de risco estimada em 1.500.000 pessoas (OPAS, 2007; WHO, 2008; 2010; 2011).

Considerada como uma das doenças potencialmente elimináveis, a OMS lançou, em 1997, o Plano Global de Eliminação da Filariose Linfática (PGEFL), que visa à eliminação da FL do planeta até 2020. As estratégias fundamentais são o tratamento em massa das populações residentes nas áreas endêmicas com drogas antifilariais e a assistência aos portadores de morbidade, principalmente linfedema e hidrocele (WHO, 1997; 1998).

Signatário do PGEFL, o Brasil elaborou o Programa Nacional de Eliminação da Filariose Linfática (PNEFL) (BRASIL, 1997; 2000; 2009), cujas atividades estão vinculadas a um grupo de trabalho fundado em 1986, no Departamento de Parasitologia do Centro de Pesquisas Aggeu Magalhães (CPqAM) - Fundação Oswaldo Cruz (FIOCRUZ), atualmente denominado como Serviço de Referência Nacional em Filarioses (SRNF). Produzindo conhecimento técnico-científico sobre esta enfermidade, em março de 2008 este serviço foi habilitado pelo Ministério da Saúde (MS) por meio da Portaria $n^{\circ} 97$ de 23 outubro de 2008 (Marcondes, 2010; BRASIL, 2008; Rocha et al., 2009). 
O SRNF desenvolve atividades interdisciplinares nas áreas clínica, epidemiológica e laboratorial, relativas à pesquisa, à assistência, à investigação e ao acompanhamento de pacientes acometidos por filarioses. O serviço presta ainda assessoria aos municípios por meio do monitoramento e controle dos casos positivos e da morbidade filarial (Marcondes, 2010; Rocha et al., 2009).

Considerando a importância do SRNF no controle da infecção filarial e a escassez de informações sobre o perfil dos pacientes assistidos por este serviço, o presente estudo foi realizado com o objetivo de descrever as características epidemiológicas desta população. O conhecimento deste perfil possibilitará o desenvolvimento de ações que promovam o aprimoramento e a melhoria da qualidade da assistência prestada.

\section{METODOLOGIA}

Foi realizado um estudo transversal descritivo, observacional, cuja população de estudo foi constituída por todos os pacientes registrados no banco de dados do SRNF, atendidos no período de 2002 a 2008. Este estudo foi aprovado pelo Comitê de Ética do Centro de Pesquisas Aggeu Magalhães - CAAE n ${ }^{\circ}$ 0005.0.095.000-10.

As informações coletadas dos prontuários dos pacientes incluíam as seguintes variáveis: idade, gênero, estado civil, procedência, autoctonia, encaminhamento médico do indivíduo, uso e tipo de medida profilática, tratamento prévio, presença de manifestação clínica e resultado da investigação realizada pela técnica parasitológica da filtração de sangue em membrana de policarbonato, dos testes para pesquisa de antígenos filarial circulante $\mathrm{Og} 4 \mathrm{C} 3 \mathrm{e} \mathrm{ICT}$ e da ultrassonografia para pesquisa de vermes adultos. Os dados obtidos foram analisados com o auxílio do pacote estatístico Epiinfo, versão 6.04 (Center for Disease Control and Prevention, Atlanta, GA), sendo utilizada a estatística descritiva para a determinação das variáveis estudadas (Dennis \& Dean, 1996) quanto à sua frequência.

\section{RESULTADOS}

No período avaliado, foram atendidos pelo SRNF 1.109 pacientes. Destes, $972(87,6 \%)$ chegaram até o serviço por encaminhamento médico. Do total de indivíduos examinados, 947 (85,4\%) eram oriundos da Região Metropolitana do Recife (RMR). A análise estratificada deste grupo permitiu identificar o município de Jaboatão dos Guararapes como a área de maior frequência de indivíduos infectados (43,9\%), seguido por Recife (37,8\%) e Olinda (11,6\%) (Tabela 1). Neste grupo, 55\% (610/1.109) dos pacientes atendidos foram diagnosticados positivos segundo pelo menos um dos métodos diagnósticos empregados para avaliação, sendo autóctones 64,8\% (247/610) dos casos. Apenas 15,2\% (168/1.109) deles relataram ter recebido tratamento prévio para filariose (Tabela 1). 
Tabela 1. Perfil dos pacientes atendidos no Serviço de Referência Nacional em filarioses, segundo procedência, autoctonia e tratamento prévio

\begin{tabular}{|c|c|c|c|c|c|c|}
\hline \multirow{2}{*}{$\begin{array}{l}\text { Variáveis } \\
\text { Procedência }\end{array}$} & \multirow{2}{*}{$\mathrm{N}$} & \multirow{2}{*}{$\%$} & \multicolumn{2}{|c|}{ Positivos* } & \multicolumn{2}{|c|}{ Negativos } \\
\hline & & & $\mathrm{N}$ & $\%$ & $\mathrm{~N}$ & $\%$ \\
\hline Recife & 457 & 41,2 & 231 & 37,9 & 226 & 45,3 \\
\hline Olinda & 124 & 11,2 & 71 & 11,6 & 53 & 10,6 \\
\hline Jaboatão & 349 & 31,5 & 268 & 43,9 & 81 & 16,2 \\
\hline Paulista & 17 & 1,5 & 2 & 0,3 & 15 & 3,0 \\
\hline Áreas não endêmicas & 112 & 10,1 & 9 & 1,5 & 103 & 20,6 \\
\hline Ignorado & 50 & 4,5 & 29 & 4,8 & 21 & 4,2 \\
\hline Total & 1.109 & & 610 & & 499 & \\
\hline Autoctonia & $\mathrm{N}$ & $\%$ & $\mathrm{~N}$ & $\%$ & $\mathrm{~N}$ & $\%$ \\
\hline Sim & 381 & 34,4 & 247 & 64,8 & 134 & 35,2 \\
\hline Não & 470 & 42,4 & 210 & 44,7 & 260 & 55,3 \\
\hline Ignorado & 258 & 23,2 & 153 & 59,3 & 105 & 40,7 \\
\hline Total & 1.109 & & 610 & & 499 & \\
\hline Tratamento prévio* & $\mathrm{N}$ & $\%$ & $\mathrm{~N}$ & $\%$ & $\mathrm{~N}$ & $\%$ \\
\hline Sim & 168 & 15,2 & 88 & 52,4 & 80 & 47,6 \\
\hline Não & 505 & 45,5 & 295 & 58,4 & 210 & 41,6 \\
\hline Ignorado & 436 & 39,3 & 227 & 52,1 & 209 & 47,9 \\
\hline Total & 1.109 & & 610 & & 499 & \\
\hline
\end{tabular}

*Tratamento prévio: Foi tratado com Citrato de Dietilcarbamazina antes de seu ingresso no SRNF.

Em relação ao gênero, 37,2\% (412/1.109) eram do feminino e 62,8\% (697/1.109) do masculino. Dos 697 homens, 60\% (418/697) mostraram-se positivos para infecção filarial. Entre eles, 25,6\% (284/1.109) declararam ser solteiros e $24,4 \%$ (271/1.109) informaram ser casados. A idade para ambos os gêneros variou de 1 a 83 anos, apresentando uma média de $32 \pm 10,25$ anos. Porém, o percentual mais elevado de pacientes atendidos - 27,9\% (309/1.109) - concentrou-se na faixa etária dos 18 aos 28 anos, que engloba 67\% (207/309) de casos positivos (Tabela 2).

Tabela 2. Perfil dos pacientes atendidos no Serviço de Referência Nacional em Filarioses, segundo gênero e faixa etária

\begin{tabular}{|c|c|c|c|c|c|c|}
\hline \multirow{2}{*}{$\begin{array}{l}\text { Variáveis } \\
\text { Gênero }\end{array}$} & \multirow{2}{*}{$\mathrm{N}$} & \multirow{2}{*}{$\%$} & \multicolumn{2}{|c|}{ Positivos* } & \multicolumn{2}{|c|}{ Negativos } \\
\hline & & & $\mathrm{N}$ & $\%$ & $\mathrm{~N}$ & $\%$ \\
\hline Feminino & 412 & 37,2 & 192 & 46,6 & 220 & 53,4 \\
\hline Masculino & 697 & 62,8 & 418 & 60,0 & 279 & 40,0 \\
\hline Total & 1.109 & & 610 & & 499 & \\
\hline \multicolumn{7}{|c|}{ Faixa etária (anos) } \\
\hline$<18$ & 261 & 23,5 & 189 & 72,4 & 72 & 27,6 \\
\hline $18 \mid-29$ & 309 & 27,9 & 207 & 67,0 & 102 & 33,0 \\
\hline $29 \mid-45$ & 293 & 26,4 & 144 & 49,1 & 149 & 50,8 \\
\hline$>45$ & 246 & 22,2 & 70 & 28,5 & 176 & 71,5 \\
\hline Total & 1.109 & & 610 & & 499 & \\
\hline
\end{tabular}

Nota: *Pacientes diagnosticados positivos segundo pelo menos uma das técnicas utilizadas (filtração de sangue em membrana de policarbonato, teste do cartão ICT, Og4C3-ELISA e/ou ultrassonografia). 
Quanto às manifestações clínicas, 48,5\% (538/1.109) dos indivíduos narraram alguma forma sugestiva de filariose linfática e as patologias mais referidas foram: linfangiectasia, varicocele e hidrocele. Entre os queixosos, 49,8\% (268/538) revelaram-se positivos em pelo menos um dos métodos diagnósticos (Tabela 3 ).

Tabela 3. Perfil dos pacientes atendidos no Serviço de Referência Nacional em Filarioses, segundo presença de manifestação clínica

\begin{tabular}{|c|c|c|c|c|c|c|}
\hline Variável & \multirow{2}{*}{$\mathrm{N}$} & \multirow{2}{*}{$\%$} & \multicolumn{2}{|c|}{ Positivos* } & \multicolumn{2}{|c|}{ Negativos } \\
\hline Presença de manifestação clínica & & & $\mathrm{N}$ & $\%$ & $\mathrm{~N}$ & $\%$ \\
\hline Sim & 538 & 48,5 & 268 & 49,8 & 270 & 50,2 \\
\hline Não & 191 & 17,2 & 123 & 64,4 & 68 & 35,6 \\
\hline Ignorado & 380 & 34,3 & 219 & 57,6 & 161 & 42,4 \\
\hline Total & 1.109 & & 610 & & 499 & \\
\hline
\end{tabular}

Nota: *Pacientes diagnosticados positivos segundo pelo menos uma das técnicas utilizadas (filtração de sangue em membrana de policarbonato, teste do cartão ICT, Og4C3-ELISA e/ou ultrassonografia).

Quanto ao questionamento referente ao uso de pelo menos uma medida profilática, apenas 31,8\% (353/1.109) desses indivíduos tinham essa informação registrada e mais da metade deles - 66,8\% (756/1.109) - não informaram qualquer dado referente a esta variável. Todavia, nos 353 indivíduos que responderam sim para profilaxia, obteve-se um percentual de 69,4\% (245) de casos positivos (Tabela 4). As medidas profiláticas mais referidas foram ventilador (13,4\%), mosquiteiro (7,8\%), inseticida (6,0\%) e lençol $(4,6 \%)$.

Tabela 4. Perfil dos pacientes atendidos no Serviço de Referência Nacional em Filarioses, segundo profilaxia

\begin{tabular}{|c|c|c|c|c|c|c|}
\hline $\begin{array}{c}\text { Variável } \\
\end{array}$ & \multirow{2}{*}{$\mathrm{N}$} & \multirow{2}{*}{$\%$} & \multicolumn{2}{|c|}{ Positivos* } & \multicolumn{2}{|c|}{ Negativos } \\
\hline Profilaxia & & & $\mathrm{N}$ & $\%$ & $\mathrm{~N}$ & $\%$ \\
\hline Sim & 353 & 31,8 & 245 & 69,4 & 108 & 30,6 \\
\hline Não & 15 & 1,4 & 12 & 80 & 3 & 20 \\
\hline Ignorado & 741 & 66,8 & 353 & 47,6 & 388 & 52,4 \\
\hline Total & 1.109 & & 610 & & 499 & \\
\hline
\end{tabular}

Nota: *Pacientes diagnosticados positivos segundo pelo menos uma das técnicas utilizadas (filtração de sangue em membrana de policarbonato, teste do cartão ICT, Og4C3-ELISA e/ou ultrassonografia).

Na Tabela 5, foi observada a positividade de cada técnica diagnóstica empregada isoladamente. A filtração de sangue em membrana de policarbonato, exame adotado como padrão-ouro pela OMS, detectou 358 indivíduos que apresentavam formas embrionárias do verme no sangue.

Analisado o total de positivos segundo gênero e faixa etária, verificou-se uma frequência quase três vezes maior, 37,4\% (261), de casos positivos na população masculina em relação a 23,5\% (97) na população feminina (Tabela 6). 
Tabela 5. Valores de resultados positivos para filtração de sangue em membrana de policarbonato, teste de cartão ICT, Og4C3-ELISA e ultrassonografia realizados no Serviço de Referência Nacional em Filarioses

\begin{tabular}{lccc}
\hline \multicolumn{1}{c}{$\begin{array}{c}\text { Resultado da investigação } \\
\text { de acordo com a técnica diagnóstica }\end{array}$} & $\begin{array}{c}\text { Exames } \\
\text { realizados }\end{array}$ & $\begin{array}{c}\text { Exames } \\
\text { positivos }\end{array}$ & $\%$ \\
\hline $\begin{array}{l}\text { Filtração de sangue } \\
\text { em membrana de policarbonato }\end{array}$ & 1.109 & 358 & 32,3 \\
\hline Teste do cartão ICT & 438 & 224 & 51,1 \\
\hline Og4C3-ELISA & 724 & 387 & 53,4 \\
\hline Ultrassonografia & 311 & 143 & 46,0 \\
\hline
\end{tabular}

Tabela 6. Distribuição segundo gênero e faixa etária dos pacientes atendidos no Serviço de Referência Nacional em Filarioses

\begin{tabular}{lcrcccccc}
\hline \multirow{2}{*}{ Faixa etária (anos) } & \multirow{2}{*}{$\mathrm{N}$} & \multicolumn{3}{c}{ Feminino } & & \multicolumn{3}{c}{ Masculino } \\
\cline { 3 - 5 } \cline { 8 - 10 } & & $\mathrm{N}$ & Positivos* & $\%$ & & $\mathrm{~N}$ & Positivos & $\%$ \\
\hline$<18$ & 261 & 96 & 19 & 19,8 & & 165 & 45 & 27,3 \\
\hline $18-29$ & 309 & 102 & 32 & 31,4 & & 207 & 96 & 46,4 \\
\hline $29-45$ & 293 & 134 & 29 & 21,6 & & 159 & 85 & 53,4 \\
\hline$\geq 45$ & 246 & 80 & 17 & 21,2 & & 166 & 35 & 21,1 \\
\hline Total & 1.109 & 412 & 97 & 23,5 & & 697 & 261 & 37,4 \\
\hline
\end{tabular}

Nota: *Pacientes diagnosticados positivos segundo pelo menos uma das técnicas utilizadas (filtração em membrana de policarbonato, teste do cartão ICT, Og4C3-ELISA e/ou ultrassonografia).

\section{DISCUSSÃO}

Os resultados encontrados apontaram a RMR como área endêmica de maior demanda de pacientes $(85,4 \%)$. Tal achado já era esperado visto que, há anos, diversos estudos vêm relatando a presença da filariose linfática no estado de Pernambuco, caracterizando-se como um problema de saúde pública na capital, Recife, e em certas cidades de sua região metropolitana (Rachou, 1957; Maciel et al., 1994; 1996; Braga et al., 1997; 2004; 2005; Bonfim et al., 2003; Brasil, 2000; Medeiros et al., 1992,1999; Vieira, 2003).

Neste trabalho, foram considerados positivos para $W$. bancrofti os indivíduos que se mostraram positivos em uma ou mais técnicas de diagnóstico utilizadas. Ficou evidenciado que mais da metade dos casos positivos $(64,8 \%)$ eram autóctones. É importante que esses indivíduos moradores de áreas consideradas de risco para infecção filarial recebam maiores cuidados, visto que estudos já demonstraram que o tempo de permanência em área endêmica influencia diretamente na aquisição da infecção filarial (Lima, 2007; Fontes et al., 1998).

Entre os positivos, muitos pacientes, na maioria homens $(55,4 \%)$, queixavam-se de alguma manifestação clínica sugestiva de filariose linfática como linfangiectasia, varicocele e hidrocele. Esta última merece especial atenção porque 
tem sido investigada como um importante indicador clínico de infecção filarial em áreas endêmicas da parasitose (De Vries, 2002).

Bonfim et al. (2003), em um estudo realizado no município de Jaboatão dos Guararapes, mostraram que entre os portadores da filariose linfática que apresentavam manifestação clínica, $69,2 \%$ eram do sexo masculino. Dreyer \& Norões (1997), estudando a morbidade filarial, estimaram que cerca de $15 \%$ dos indivíduos infectados desenvolverão alguma forma clínica da doença filarial.

$\mathrm{Na}$ análise por idade, foi evidenciada maior positividade entre os indivíduos na faixa etária abaixo dos 18 anos (72,4\%). Este resultado contrasta com estudos realizados por Sasa et al. (1976) e Maciel et al. (1994) que apontam a faixa etária pediátrica como responsável pelas menores taxas de prevalência. No entanto, deve-se ressaltar que esses trabalhos utilizaram como ferramenta diagnóstica exclusivamente o método parasitológico da gota espessa de sangue. Esta técnica já é tida como inapropriada na investigação de populações pediátricas caracterizadas por apresentarem baixas microfilaremias ou serem amicrofilarêmicas.

Quando analisados exclusivamente os casos positivos pelo método parasitológico, observou-se que a população masculina apresentou uma frequência de casos positivos quase três vezes maior do que a população feminina. E mais, que a faixa etária mais atingida entre os homens compreendeu o intervalo dos 18 aos 44 anos de idade.

Estes achados corroboram os dados da literatura que evidenciam ser a população masculina em idade economicamente ativa não só a mais atingida pela infecção filarial, mas também a que tem mais chances de adquiri-la (Lima, 2007; Rocha et al., 2000). Este quadro pode, em muitos casos, incapacitar esses indivíduos para o trabalho e comprometer seu desenvolvimento econômico e social (Dreyer et al., 1997; Fontes, 1996; Rocha, 2004).

Com relação à utilização de medidas profiláticas, o índice foi considerado baixo, porém esta variável pode encontrar-se subestimada. Todavia, o baixo índice de profilaxia não surpreende os pesquisadores uma vez que os indivíduos acometidos por esta parasitose residem em áreas onde predominam precárias condições sanitárias e, em geral, eles apresentam baixo poder socioeconômico, o que torna inviável a utilização de tais medidas. Em estudos realizados em um serviço que presta assistência aos portadores de filariose linfática, Mattos e colaboradores (2008) apontam as baixas condições socioeconômicas em que vivem crianças parasitadas e seus familiares.

Outro dado que chama a atenção é o percentual de $15,2 \%$ de indivíduos que relataram uso de tratamento prévio antifilarial específico. Acredita-se que este dado também se encontre subestimado em razão da ausência significativa de informações referentes a esta variável. Em levantamentos futuros, esta taxa deve ser superada já que o tratamento coletivo tem sido implantado em áreas endêmicas de filariose no estado de Pernambuco. Com o avanço da área de cobertura do tratamento coletivo, espera-se reduzir a taxa de transmissão da $W$. bancrofti e, assim, contribuir para o PGEFL. 
Diante do observado, o presente estudo conclui que o perfil apresentado pelos pacientes encaminhados ao Serviço de Referência Nacional em Filarioses corrobora os achados relatados na literatura. Estas informações viabilizaram a elaboração de novos algoritmos clínico-administrativos, melhorando a qualidade das atividades desenvolvidas pelo serviço.

\section{AGRADECIMENTOS}

Agradecemos o apoio financeiro do SRNF/Departamento de Parasitologia/CPqAM em parceria com a FIOTEC/SVS e FIOCRUZ/SVS. Estendemos nossos agradecimentos a todos que direta ou indiretamente colaboraram ou colaboram com as atividades desenvolvidas no serviço, possibilitando uma assistência cada vez melhor aos nossos pacientes.

\section{REFERÊNCIAS}

1. Braga C, Albuquerque MFM, Schindler H, Rezende A, Maciel A, Silva MCM, Furtado A, Carvalho AB, Lapa T, Ximenes RAA. Perfil Epidemiológico da filariose linfática em crianças residentes em áreas endêmicas. Jornal de Pediatria 73: 95-100, 1997.

2. Braga C, Albuquerque MFM, Morais HM. A produção do conhecimento científico e as políticas de saúde pública: reflexões a partir da ocorrência da filariose na cidade do Recife, Pernambuco, Brasil. Cad Saude Publica 20: 351-361, 2004.

3. Braga C, Dourado I, Ximenes R, Miranda J, Alexander N. Bancroftian filariasis in an endemic area of Brazil: differences between genders during puberty. Rev Soc Bras Med Trop 38: 224228, 2005.

4. Brasil, Ministério da Saúde. Coordenação de Controle de Doenças Transmitidas por vetores. The Filariasis Elimination Program: Urban Challenges, Focal Elimination and Its Verification. In: Programa de Eliminação da Filariose Linfática no Brasil, Brasília, 1997.

5. Brasil (Fundação Nacional de Saúde). Relatório da Reunião de Avaliação do Programa de Controle da Filariose Linfática, referente ao Plano de Eliminação da Filariose Linfática no Brasil, Recife - PE. Brasília, Brasil, 2000.

6. Brasil, Ministério da Saúde. Portaria 97, de 23 de outubro de 2008. Habilita Laboratórios de Referência Nacional para as Redes Nacionais e Regionais de Laboratórios de Vigilância Epidemiológica. Diário Oficial da União, Brasília, DF, Secção 1, 2008, p.51.

7. Brasil. Secretaria de Vigilância em Saúde. Departamento de Vigilância Epidemiológica. Guia de vigilância epidemiológica e eliminação da filariose linfática, Brasília, 2009.

8. Bonfim C, Lessa F, Oliveira CO, Evangelista MJ, do Espirito Santo M, Meireles E, Pereira JC, Medeiros Z. The occurrence and distribution of lymphatic filariasis in Greater Metropolitan Recife: the case of an endemic area in Jaboatão dos Guararapes, Pernambuco, Brazil. Cad Saude Publica 19: 1497-1505, 2003.

9. Dennis DT, Dean AG. Epi Info, version 6.0b: a word processing database and statistical program for epidemiology on microcomputer. Stone Mountain: Centers for Disease Control, 1996.

10. De Vries CR. The role of the urologist in the treatment and elimination of lymphatic filariasis worldwide. BJU International 81: 37-43, 2002.

11. Dreyer G, Norões J. Dietilcarbamazina no Tratamento da Filariose Bancroftiana. Rev Soc Bras Med Trop 30: 229-240, 1997.

12. Fontes G. Aspectos epidemiológicos da filariose linfática causada pela Wuchereria bancrofti no Estado de Alagoas. Belo Horizonte [Tese de Doutorado - Departamento de Parasitologia do ICB /UFMG], 1996.

13. Fontes G, Rocha EMM, Brito AC, Antunes CMF. Lymphatic Filariasis in Brazilian Urban Area (Maceió, Alagoas). Mem Inst Oswaldo Cruz 93: 705-710, 1998.

14. Fontes G, Braun RF, Neto HF, Vieira JBF, Padilha SS, Rocha RC, Rocha EMM. Filariose linfática em Belém, Estado do Pará, Norte do Brasil e a perspectiva de eliminação. Rev Soc Bras Med Trop 38: 131-136, 2005. 
15. Lima ARV. Situação epidemiológica da filariose linfática no foco endêmico de MaceióAlagoas após a implantação do programa de eliminação. Maceió [Dissertação de Mestrado em Ciências da Saúde - UFAL], 2007.

16. Maciel A, Marzochi KBF, Silva EC, Rocha A, Furtado AF. Estudo comparativo de áreas endêmicas de filariose bancroftiana na Região Metropolitana do Recife, Brasil. Cad Saúde Pública 10: 301-309, 1994.

17. Maciel A, Rocha A, Marzochi KBF, Medeiros Z, Carvalho AB, Regis L, Souza W, Lapa T, Furtado A. Epidemiological Study of Bancroftian Filariasis in Recife, Northeastern Brazil. Mem Inst Oswaldo Cruz 91: 449-455, 1996.

18. Marcondes MMC. Conhecimento e Práticas em portadores de morbidade filarial linfática no município de Olinda-PE, Brasil. Recife [Dissertação de Mestrado em Saúde Pública - Centro de Pesquisas Aggeu Magalhães / FIOCRUZ], 2010.

19. Mattos D, Mota S, Dreyer G. Aspectos da realidade social de crianças e adolescentes atendidos em serviço de referência para filariose bancroftiana - Recife, Estado de Pernambuco. Rev Soc Bras Med Trop 41: 29-35, 2008.

20. Medeiros Z, Dreyer G, Andrade LD, Pires ML, Mendes J, Pimentel R. Wuchereria bancrofti microfilarial density of autochthonous cases and natural Culex infectivity in Northeast Brazil. J Trop Med Hyg 95: 214-217, 1992.

21. Medeiros Z, Gomes J, Béliz F, Coutinho A, Dreyer P, Dreyer G. Screening of army soldiers for Wuchereria bancrofti infection in the Metropolitan Recife Region, Brazil: implications for epidemiological surveilance. Trop Med Int Health 4: 499-505, 1999.

22. Rachou RG. Distribuição geográfica das filarioses humanas no Brasil. Rev Bras Malariol Doenças Trop 9: 79-100, 1957.

23. Rey L. Wuchereria bancrofti e filarioses linfáticas. In: Parasitologia: Parasitos e Doenças parasitárias do homem nas Américas e na África. Guanabara Koogan. Rio de Janeiro, 2001.

24. Rocha A. A Filariose bancroftiana: Avaliação dos testes diagnósticos frente às diversas formas clínicas da bancroftose. Rio de janeiro [Tese de Doutorado em Biologia Celular e Molecular - Instituto Oswaldo Cruz, Fundação Oswaldo Cruz], 2004.

25. Rocha A, Barbosa CS, Brandão Filho SP, Oliveira CMF, Almeida AMP, Gomes YM. Primeiro workshop interno dos serviços de referência do Centro de Pesquisas Aggeu Magalhães da Fundação Oswaldo Cruz. Rev Soc Bras Med Trop 42: 228-234, 2009.

26. Rocha EMM, Fontes G, Brito AC, Silva TRC, Medeiros Z, Antunes CMF. Filariose bancroftiana em áreas urbanas do estado de alagoas, nordeste do Brasil: estudo em população em geral. Rev Soc Bras Med Trop 33: 545-551, 2000.

27. Sasa M. Human Filariasis - A Global Survey of Epidemiological and Control. University of Tokyo Press, Japão, 1976.

28. WHO (World Health Organization). Elimination of lymphatic filariasis as a public health problem. Fiftieth World health Assembly, Geneva, 1997.

29. WHO (World Health Organization). Guidelines for certifying lymphatic filariasis elimination. Lymphatic Filariasis Eliminate Programme. Geneva, 1998.

30. WHO (World Health Organization). Global Programme to Eliminate Lymphatic Filariasis: Progress report on mass drug administration in 2007. Wkly epidemiol Rec 83: 333-348, 2008.

31. WHO (World Health Organization). Global Programme to Eliminate Lymphatic Filariasis. Progress report 2000-2009 and strategic plan 2010-2020 of the global programme to eliminate lymphatic filariasis: halfway towards eliminating lymphatic filariasis, Geneva. 2010.

32. WHO (World Health Organization). Global Programme to Eliminate Lymphatic Filariasis. Monitoring and epidemiological assessment of mass drug administration in the global programme to eliminate lymphatic filariasis: a manual for national elimination programmes. World Health Organization. Geneva. 2011.

33. OPAS (Organização Pan Americana de Saúde). Condições de Saúde e suas tendências. In: Saúde nas Américas. Washington, 2007, v. 1, p. 62-217. OPAS, 2007.

34. Vieira JBF. Brazil: the filariasis elimination program in Brasil: current status, challenges, and roads to the future. In: Regional Program Manager's Meeting, 2003, Maceió, $4^{\text {th }}$ regional program manager's meeting: lymphatic filariasis elimination in the Americas. Maceió: Pan American Health Organization, 2003. p 13-32. 